\title{
in Technology
}

\section{Research Paper}

\section{The Role of MEMS in In-Vitro-Fertilization}

\section{D. Wijegunawardana a, ${ }^{*}$ and Y. W. R. Amarasinghe ${ }^{b, c}$}

a Department of Materials and Mechanical Technology, University of Sri

Jayewardenepura, Pitipana, Homagama 10200, Sri Lanka

${ }^{b}$ Department of Mechanical Engineering, University of Moratuwa, Katubadda 10400, Sri Lanka

c Centre for Advanced Mechatronic Systems (CFAMS), University of Moratuwa, Katubadda 10400, Sri Lanka

Email correspondence: isiranc94@gmail.com (I. D. Wijegunawardana)

Received: 21 March 2021; Revised: 28 April 2021; Accepted: 23 May 2021; Published: 31 May 2021

\begin{abstract}
The assisted reproduction has been considered a viable solution for the infertility of humankind for more than four decades. In-Vitro-Fertilization (IVF) is one of the most successful assisted reproduction techniques, where the reproductive cell of the female partner is fertilized outside of her body. Initially, the IVF process has been conducted manually by an experienced embryologist. However, even with a highly experienced individual, the operation had extremely lower success rates due to the limited control in environmental conditions and the requirement of precise movements. Therefore, to address this technological deficit, the feasibility of the mechatronics devices for IVF procedures has been investigated. Among the different mechatronics concepts, microelectromechanical system (MEMS) technologies have been gradually attracted to the IVF process and improved its capabilities. The purpose of this paper is to present a brief overview of the role of MEMS technologies in IVF. The article classifies the MEMS technologies in IVF based on their application in order to emphasize its contribution. In addition, the article extensively discusses the state-of-the-art mechatronic techniques utilized in Intracytoplasmic Sperm Injection (ICSI), one of the most popular techniques used in IVF. This review article expects to become extremely beneficial for the engineering researchers new to this field who seek critical information on IVF in simple terms with highlights on the possible advancements and challenges that may emerge in the future.
\end{abstract}

Keywords: Assistive-reproduction, in-vitro-fertilization, ICSI, mechatronics, MEMS 


\section{Introduction}

The incompetence of reproduction has been a striking issue for mankind for years. One of the main reasons for this: the infertility of male or female companion, has been resolved by introducing Assisted Reproductive Technology (ART). ART is a highly advanced medical discipline that consists of three major branches (See Figure 1). Among these ART treatments, In-Vitro Fertilization (IVF) is a popular technique employed where both woman's oocytes (egg) and man's spermatozoon (sperm) are handled externally, in a controlled environment to create an embryo, which will be subsequently transferred into the female companion's uterus (womb). On the other hand, in artificial insemination, the spermatozoon of the male companion is inserted into the reproductive tract of the female companion, while in the surrogacy method, the embryo is growing inside of a separate female individual (surrogacy mother) on behalf of the parents [1].

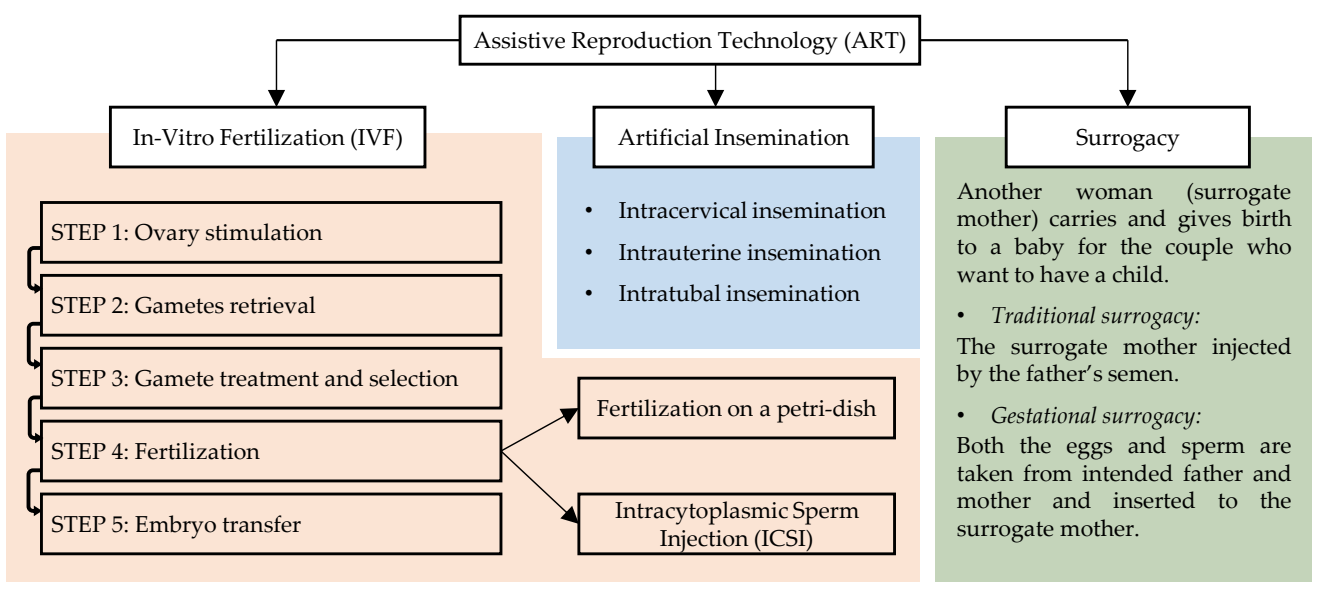

Figure 1. Classification of ART technologies and main steps of IVF

The history of IVF dates back to the 1890s, where the first known case of embryo transplantation in rabbits reported [2]. This was long before the first-ever application of IVF on human fertility was successfully conducted in 1978, and achieved the first test-tube baby, Louise Brown, in Oldham, England [3]. The IVF process consists of several steps (See Figure 1) such as ovary stimulation; retrieving the gametes; performing 
culture procedures on gametes; selecting the viable gametes for fertilization; immobilization of sperms; inserting sperms into the oocyte (insemination) to produce an embryo; continuous monitoring and evaluating embryos; and finally transferring the embryo into the mother's uterus [4]. Here, as shown in Figure 1, the fertilization process can be conducted according to two methods. They are: (1) allowing the fertilization to be occurred on a petri-dish without external intervention; and (2) forcing the sperm into the oocyte with the help of a glass pipette. The second method is commonly known as Intracytoplasmic Sperm Injection (ICSI) and considered to be a more successful technique compared to its counterpart [5].

Over time, there have been lots of developments in IVF technology and currently became a notable research area that utilizes not only medical science but also the engineering and technological disciplines [6]. MicroElectromechanical Systems (MEMS) is one such engineering domain that most probably will decide the future directions of IVF. In recent research attempts on IVF, MEMS involvements have been noticed in assisting mechanical activities such as oocyte selection, gametes conveying, sperm immobilization and sperm insertion into the oocyte [7]. Autonomous MEMS-based technologies have more control over the environmental conditions due to the lesser human involvement and prevention of becoming exposed to the air. Moreover, they have precise positioning that can reduce oocyte damages during cell handling.

In general, micromachining and micro-electromechanical system (MEMS) technologies are being used to produce devices on the scale of micrometers. The main reason for this is, in MEMS, a wide variety of transduction mechanisms can be used to convert real-world signals from one form of energy to another. These devices use mechanical, fluidic, thermal, magnetic, optical and chemical properties of different materials which are known as smart materials to employ these energy transformations. With these properties, smart materials have been used in various micro devices such as sensors, actuators, energy harvesters, 
microfluidic systems, so on and so forth. (See Figure 2) This ability of miniaturization of devices and systems has offered a greater opportunity to achieve commercial profit in many fields such as telecommunication, transportation, construction, military, healthcare and so forth [11].

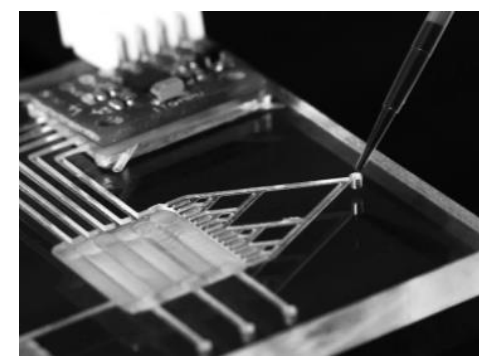

(a)

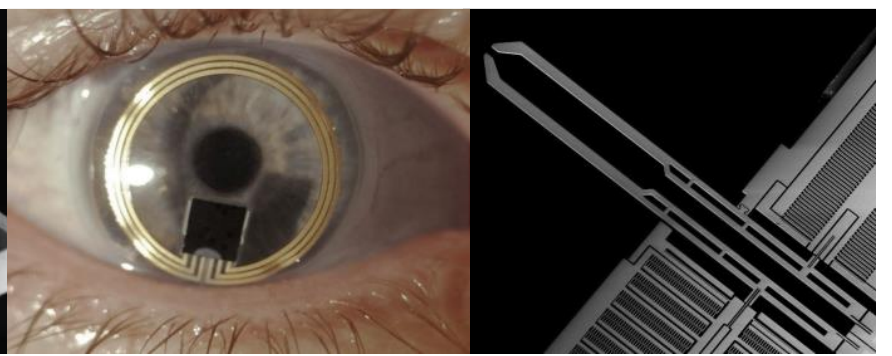

(b) (c)

Figure 2. Commercially available MEMS devices: (a) a microfluidic device [8], (b) a contact lens sensor for glaucoma patients [9], (c) an electrostatically actuated micro gripper developed for micro scale manipulation [10]

On the other hand, MEMS technology has become a trending branch of biomedical engineering which is known as Bio-MEMS [12]. Bio-MEMS concepts can be identified in several biomedical applications such as drug delivery [13], medical surgeries [14], energy harvesting, medical monitoring [See Figure 2(b)], cell manipulation [15] and so forth. Furthermore, the novel concept of lab-on-a-chip devices has immensely contributed to Bio-MEMS. However, incredible precisions, efficiency and biocompatibility of MEMS transducers present in Bio-MEMS applications have shown positive signs for potential achievements in IVF related applications. In order to accomplish those achievements, the enhancement of the research interest of MEMS researchers on IVF is necessary. For instance, the article [16] investigates the research involvement in automating assisted reproductive technology and state that still there is a long way to go before ART procedures can be automated in one single device. However, during the analysis on research that intersect MEMS and IVF, it was evident that articles that summarise the previous research attempts were extremely hard to find. 
The brief overview provided in this paper has summarized the MEMSbased mechatronics techniques used in IVF providing classifications to emphasize their contribution. The upcoming sections of this paper comprise MEMS involvement in different stages of IVF. In the later sections, the paper has extensively explained the role of MEMS in ICSIIVF, since, in recent times, ICSI has broadly investigated by the BioMEMS research community. As a final point, the paper explains the possible future advancements and challenges in this research area.

\section{MEMS Involvement in IVF}

In the literature, an increasing trend can be seen in MEMS usage in microbiological applications [12] where IVF is one such application that recently came to the forefront. During the analysis for the MEMS-based developments in IVF, several research attempts were found assisting the IVF process in its different steps. The following subsections explain the MEMS involvement in IVF classified based on the application. Figure 3 provides a brief overview of the selected scope for this article and how it is branched out to IVF and MEMS technologies.

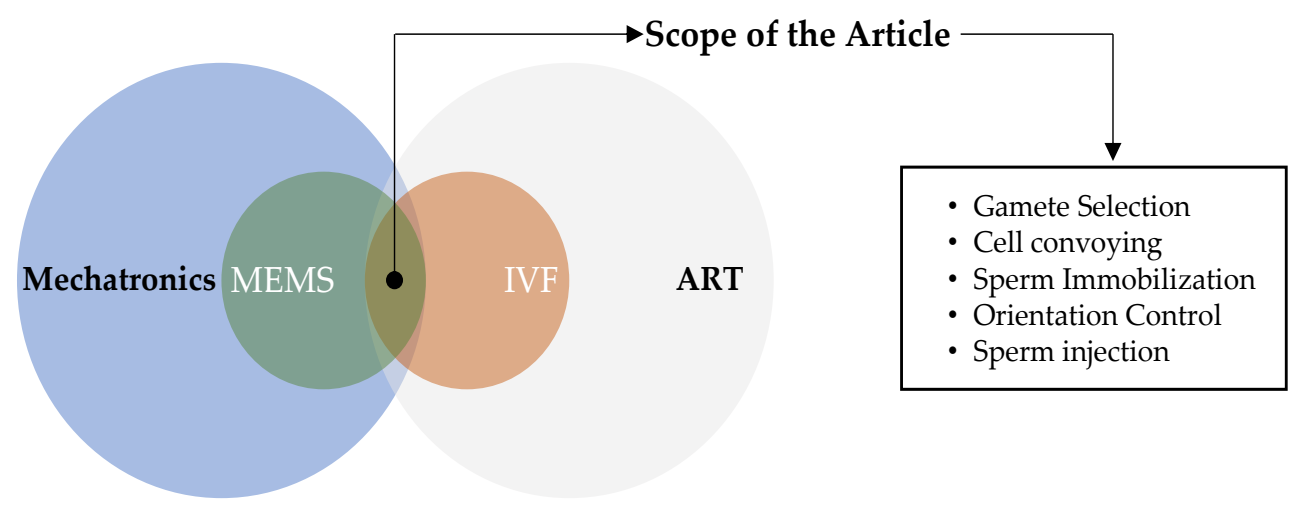

Figure 3. Scope of the article and taxonomies

\section{Gamete Selection}

The selection of the ideal sperm for IVF is based on its movability and often considered as not much complicated. Hence, in the conventional procedure selection is conducted based on the manual observation of 
sperms in the petri dish even though it does not provide the best sperm. However, recent MEMS advancements have suggested microfluidic devices for sperm sorting. Here, the motile sperms are filtered out when they swim out through the microfluidic channels present in these devices. For instance, the study published in [17] has assessed the ability of sperm to swim through laminar interfaces. With further improvements, [18] has proposed a method to control the viscosity of the fluid in the device where the environment of the microfluidic device can be made similar to the human body, so that, the selection can be more precise. Even though these methods are able to select more motile sperms, due to the less controllability of the microfluidic flows, still these devices are not as efficient as the manual selection process.

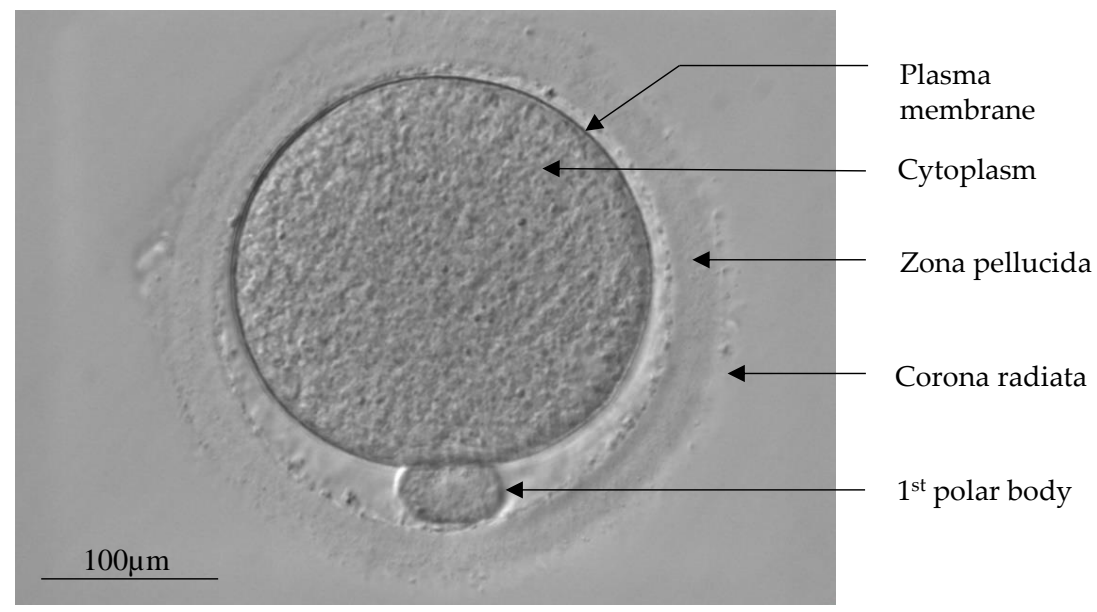

Figure 4. Cell morphology of an MII stage oocyte [19]

Nevertheless, the selection/sorting of the Oocyte is considered one of the most critical steps in the IVF process since its progress entirely depends on the health of the oocyte. The most suitable oocyte for the IVF is identified as the metaphase II (MII) stage which appears during the oocyte growth after the retrieval [4]. The identification of this oocyte is conducted by morphological observation. The general configuration of an MII stage oocyte is shown in Figure 4. However, with extensive research on the successfulness of these MII stage oocytes, it has been 
concluded that even this MII stage show significant morphological variations that may affect the developmental competence and implantation potential of the derived embryo [4].

On account of the critical requirement, a variety of techniques have been introduced for the assessment of ideal oocyte [20]. Among them most of the methods are based on chemicals and observation. Hence, they take a large time to provide an accurate selection. On the other hand, there are some mechanical approaches that can be used to evaluate the oocyte by its mechanical properties such as yield strength, elasticity, and poisons ratio. In [21] MEMS-based techniques has been investigated for this purpose which suggests a force-sensing method to find the rigidity of the human oocyte cell, by which it [18] can be characterized as viable or not for the fertilization. Here, the force feedback on the cylindrical glass tip, which is controlled by a magnetic field, is assessed when it presses the oocyte. In addition, for the same purpose, [22] refers to a dielectrophoretic separation method of porcine oocytes. In this study, the oocytes were selected upon the amount of movement that showed as responses to the different dielectrophoretic conditions.

\section{Cell Conveying}

In IVF transporting the cells from one place to another also inspired some research groups. The research conducted in $[21,23,24]$ introduces a micro pusher used in positioning and conveying the oocyte. Here, the control method of the micro pusher is proposed as a magnetic field because it has untethered remotely control capabilities. Moreover, the MEMS device introduced in [25] has used the dielectrophoretic technique to develop a dynamic-minima in the surface, thus the cell is guided to the desired location. However, a common drawback of these techniques was the limitation of the manipulation of cells to only two degrees of freedoms.

Other than the structural techniques, research attempts on microfluidic approaches [26] also identified in the literature for gametes convoying. These microfluidic devices utilize pressure variations to control the fluid 
flow and convey gamete cells along with that. To stop/trap the cells, the most commonly used technique is to develop microstructures such as micro-wells. These are the key techniques generally used for lab-on-achip development [26], which is considered as the future of the bioMEMS. These devices can facilitate multiple operations in a single device. Here the micro-fluidic technologies provide the conveying of gametes from one workstation to the next. On the contrary, micro-fluidic techniques with pressure variation can be hostile for the oocytes due to their fragile nature. Moreover, these techniques have less precision and high uncertainty compared to structural manipulation.

\section{Sperm Immobilization}

Most of the male candidates have a certain proportion of motile and viable sperms. Even though this mobility is an advantage for the embryologists to easily filter the healthy sperms, they are facing the problem of aspirating the moving spermatozoon into the pipette and handling them through the procedure. For these purposes, in some instances, elements such as PVP and hyaluronate are added to the culture media, to slow down the movements of the sperm, so that it can be caught. After the spermatozoon is caught, it is immobilized before the injection to reduce the possible damages on the cytoskeleton caused by motile sperms, and to increase the chances of fertilization since the damaged sperm membrane facilitates exposure of the nucleus to the cytoplasm [27]. Sperm immobilization is performed using different methods. Even though novel methods $[28,29]$ such as laser-assisted permeabilization of the sperm membrane have been introduced, the most common approach is to press the sperm tail with the injection pipette. The common drawback seen in the current research attempts were the potential of damaging the sperm during the immobilization which leads to low success rates.

\section{Sperm Insertion}

Nevertheless, while IVF employs state-of-the-art MEMS solutions for the above applications, the current trend of sperm insertion is also moved 
towards MEMS-based techniques. Therefore, this paper pays enhanced attention to the state-of-the-art solutions for sperm insertion in IVF. This can be conducted in two methods; either by introducing the gametes on a petri dish at the laboratory letting the fertilization occur naturally, or by Intracytoplasmic sperm injection (ICSI) where a single sperm is injecting into the oocytes through a micro pipet [5]. However, the ICSI method is rather effective since sometimes in ART, the sperms are not strong enough to be mobilized. The next section of this paper extensively explains the notable developments in the ICSI procedure.

\section{MEMS Involvement in ICSI}

ICSI process has been manually conducted by an expert embryologist for years. However, manually handling micro-scale objects by a human was, for the most part, unsuccessful. Therefore, to increase the success rate of the ICSI process there has been a multitude of research conducted by several research groups. So far, several methods have discovered other than MEMS-based injection for introducing foreign materials into a cell such as viral vectors, electroporation, ultra-sonic and gene gun. However, compared to these techniques, microinjection with a single glass micropipette has found to be the most effective, since it reduces cell damage, cell waste, cell viability, specificity and phenotype alteration while increasing the effectiveness of delivering macromolecules [30]. Therefore, the automation of the ICSI process has been a trending solution that is being extensively investigated by the research community. However, to automate the ICSI process, understanding the key steps in the conventional procedure and identifying its limitations is essential.

\section{Conventional ICSI Procedure}

The conventional ICSI procedure tends to follow a common pattern. To simplify the explanation, henceforth the locations of components have described using the clock position method. 


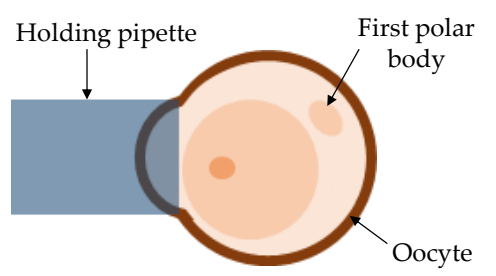

(a)

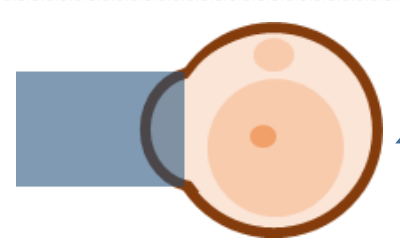

(c)

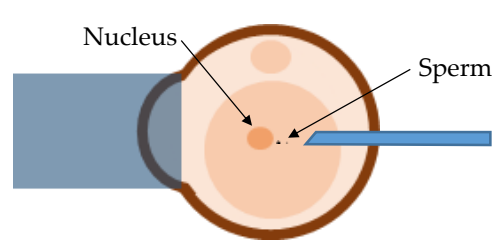

(e)

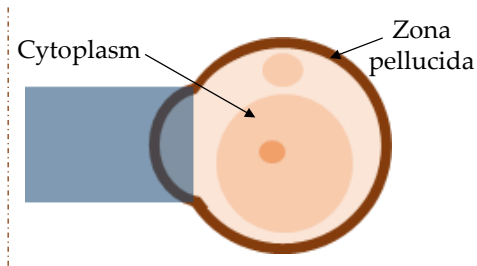

(b)

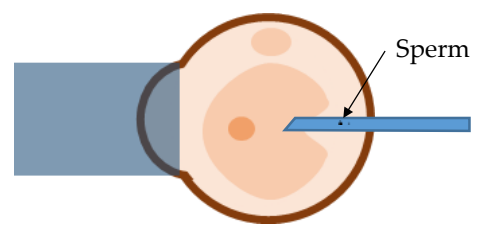

(d)

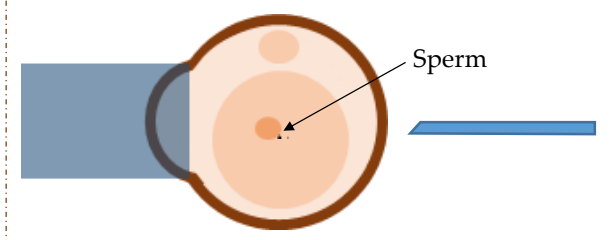

(f)

Figure 5. Conventional ICSI process, (a) Random initial orientation of the oocyte immobilized by holding pipette, (b) First polar body rotated to the 12 O'clock position, (c) taking a sperm cell into the injection pipette, (d) drilling the zona pellucida and creating a characteristic funnel, (e) releasing sperm in the approximate centre of the oocyte, (f) slowly retracting the injection pipette

Firstly, the oocyte is immobilized with the holding pipette which is located at $9 \mathrm{O}^{\prime}$ clock using suction (negative pressure) [See Figure 5(a)]. Then the oocyte is rotated slowly [31] to place the first polar body farthest from the path of the injection needle (on the 6 or $12 \mathrm{O}^{\prime}$ clock position) [See Figure 5(b)], to protect the meiotic spindle [32], which is considered to be located in the boundary of the oocyte below the first polar body from being damaged. Subsequently, the equatorial plane of the oocyte is focused, and the injection pipette will be pressed against the zona pellucida [See Figure 5(d)], creating a characteristic funnel, breaking the membrane, at $3 \mathrm{O}^{\prime}$ clock, at the approximate centre of the oocyte. Then by penetrating both the zona and the plasma membrane, a small volume of cytoplasm will be aspirated into the glass pipette to activate the oocyte and to ensure entering of the ooplasm. The single immotile spermatozoon is then gently placed near the horizontal axis 
[See Figure 5(e)]. Finally, the withdrawal is conducted carefully to prevent the oocyte from leakages [See Figure 5(f)]. However, to avoid leaving behind residual medium with the spermatozoon and to close the breach of penetration, a mild suction is done, while removing the pipette, when the pipette is at the approximate centre of the egg, where some extra-medium is re-aspirated so that the cytoplasmic structures can envelop the sperm, thereby reducing the size of the breach. [33].

However, the conventional ICSI process contains several deficiencies which need to be rectified. In ICSI sometimes the nutritive cells might block the 9 O'clock position and the oocyte cannot be manipulated as required with the holding pipette. Moreover, in ICSI, a risk of accidentally bring in foreign somatic DNA into the oocyte is present if the site of injection has remaining attached cumulus cells. Predominantly, optimum pressure should be applied during oocyte handling since the increased pressure can damage the gamete. Thus, ICSI possesses rather higher risks of doing permanent damages on the injected oocyte such as lysis, shrinkage, and tannings which results in deteriorated oocytes at a rate between $1 \%$ to $3 \%$ [4]. Furthermore, other critical problems that emerge during the handling of spermatozoon in ICSI are: spermatozoon remaining stuck to the injection pipette while being released, spermatozoon are not being sufficiently immobilized to prevent tail movements after injection, rejecting spermatozoon into perivitelline space after injection so that the sperm's tail sticking out of the zona pellucida, and difficulties in breaking the plasma membrane.

\section{Mechatronics in ICSI Procedure}

Due to the low success rate and slow injection rate of ICSI, manual manipulation requires long training and the experience of the operator. Therefore, research on human-free robotic devices for IVF started with [34]. Thereafter, similar research on autonomous micromanipulation systems immerged in $[30,35,36]$. These systems have the most commonly used vision feedback with closed-loop control for precise control of the micro-pipettes. Currently, the research on autonomous 
micromanipulators are more focused on developing their visual surveying features [37-39]. Even so, those attempts have not shown the required levels of success in performance to be qualified for commercial use.

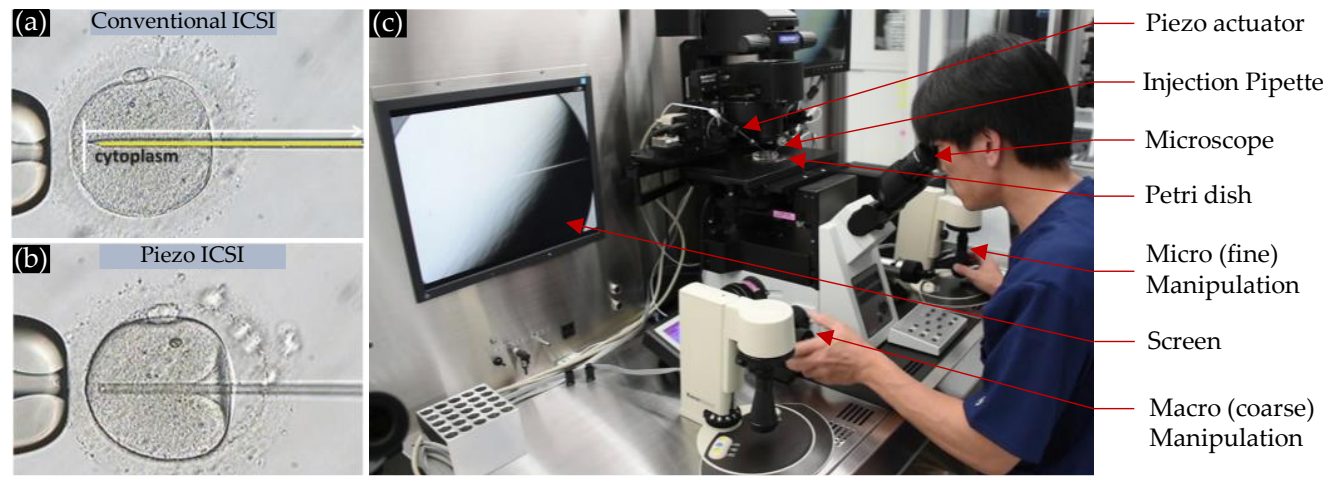

Figure 6. (a) in conventional ICSI, a large amount of cytoplasm is aspirated into the micropipette at the point of membrane breakage [4], (b) in piezo ICSI, only a little or no cytoplasm is aspirated [4], (c) A modern micromanipulation device to perform

Piezo-ICSI procedures [40]

Nevertheless, the top-notch mechatronics micromanipulation systems being used in ICSI outputs a microscale cell manipulation for a macroscale input [41,42]. These machines consist of microscopes that have a 200-400 times magnification to visualize the morphological details of the human oocytes. They also have control units, where the micropipettes are fixed in a holder that can be moved with both centimetre and micrometre precisions based on the requirement of coarse or fine movement. [See Figure 6(c)] This technology has increased the efficiency of manipulation, and at the same time increasing the accuracy.

These machines have two separate controllers with a dedicated physical interphase [43] (teleoperation) such as a joystick, a rotating knob or graphical computer interphase, for each. The centimetre manipulation will be conducted through electric motors while rather sensitive actuating techniques, such as hydraulic, pneumatic, piezoelectric, and mechanical, will be involved in the micrometre manipulation. Moreover, some ongoing research expects to provide haptic feedback (tactile 
perception) to the operator through the teleoperated machines [44-47], to increase the effectiveness of existing mechatronic systems in the future.

\section{MEMS-based Orientation Control}

During the ICSI process, the orientation of the oocyte cell plays an important role. This orientation should facilitate an obstacle-free path to the microneedle $[32,48]$ during sperm injection. In the conventional ICSI process, this is carried out by collaboratively controlling the injection and holding pipettes. However, this manual process is time-consuming and prone to damage the oocyte. As a solution for this in [49], MEMS technology has intervened in developing high precision stages with micro-wells that can be rotated with micro precision. The research described in [50] suggests MEMS-based actuation to obtain the expected orientation by rotating the oocyte at the desired angle. On the other hand, there are number of other alternatives investigated in literature for cell rotation using, microfluidic [51,52], optical [53,54], vibration [55,56], magnetic [21,56,57] and electric [58-60] methods. Even so, these techniques do not guarantee the non-destructive application on human oocytes. Furthermore, unlike structural actuators, they do not provide the accuracy for open-loop control.

\section{MEMS-based Sperm Injection}

Although the majority of researchers have attempted to develop the control aspects of the glass micropipettes, some of the research has been carried out to design and fabricate innovative microinjection tools/systems to reduce the damage that can happen to the oocytes [61,62]. The conventional borosilicate glass capillaries, produced through pipette pulling, micro-forging and grinding, has been very popular in IVF Laboratory for a long time [63] because of the ability to sanitize and sterilize on-site by the physician himself made them extremely cost-effective. However, due to the requirement of an experienced operator, the glass pipette is most probably will be overthrown by the innovative MEMS-based injectors [61,64]. In recent 
times, MEMS-based injection is much popular in drug injection and cancer cell treatments. Its invasion of the ICSI is yet to be initiated. For instance, in [64] a novel microinjector has been extensively analysed for oocyte penetration and shown promising results in terms of oocyte damage.

In recent times, the concept of piezo-ICSI has been getting increased attention in the literature. Compared to the conventional ICSI [See Figure 6(a)], the state of art technique introduced as piezo-ICSI possesses several advantages. In Piezo-ICSI, the membrane is breaking with a piezo pulse $[37,65]$, which produces ultrafast forward momentum in a submicron scale, using flat-tipped micropipettes (no bevelled or spiked tips). During zona penetration, the injection pipette can drill the zona without any deformation, and without any cytoplasm is aspirated into the micropipette during membrane breakage. [See Figure 6(b)] The ICSI can achieve a $54 \%$ fertilization rate and a $33 \%$ clinical pregnancy rate, respectively, while ICSI by piezoelectric activation can raise it to $48 \%$ and $44 \%[66,67]$.

\section{Challenges and Future Directions}

The success rate and the effectiveness of the IVF process depend upon environmental factors such as light, vibration, temperature, and humidity. For example, in ICSI, the temperature should be constantly kept at a range of $35-38{ }^{\circ} \mathrm{C}$ throughout the complete process. For this purpose, the temperature of all laboratory devices such as heating plate, transportable container, microscope stage and incubator, should be set higher than the requested temperature. Therefore, even with the autonomous control of pipette manipulators, the expected level of control cannot be provided over environmental conditions during the fertilization process since it can take up to 6 hours to complete. In addition, in the IVF-ICSI process with glass pipettes, excessive or vigorous handling of the oocyte may result in zona pellucida fracture and even oocyte degeneration. Therefore, the unexpected control 
outputs (shocks) in the automated machines should be minimized to achieve a successful outcome in the IVF process.

On the other hand, since the MEMS technologies are in their early ages, there is a notable research gap to be addressed for this field to become established. For instance, the smart material technology, that is acting as the heart of the MEMS, is highly sensitive to the environment and can be damaged easily. Therefore, for the time being, complete dependence on MEMS technology can be doubtful. Especially, due to these inadequacies, keeping the specimen at the required temperature and receiving steady movements from MEMS actuators at the same time can be challenging.

Along with the MEMS actuation and sensing, image processing can be considered as another key area that requires to be developed for the autonomous control of microdevices for IVF. Even though some of the MEMS actuators can be controlled with an open-loop control system, when the microfluidic techniques get involved in the device, feedback control is essential. Hence, the microscope image processing technology should be improved to harvest the higher success rates in MEMS devices.

Apart from the research topics highlighted in this article, in the future, ART requires improvements in the methods for gamete retrieval, embryo development and embryo insertion. Furthermore, ultimately the expectation on behalf of IVF is to develop an all-in-one robotic device with MEMS technology, which can perform all the tasks in ART including the IVF and ICSI operations without human involvement.

Nevertheless, most of the ongoing research on this topic is carried out focusing on a single step in the IVF process. Hence, the compatibility among different MEMS-based solutions is questionable when considering them in a single unit. To solve this, the intervention of review articles is required on this topic. Then, the research community can divulge their findings in a common platform where others can easily access. Hence, authors expect this review article will be contributed as a 
solution for that, and become an aid to achieve the ultimate target of developing a MEMS-based single device (lab-on-a-chip) that autonomously facilitate all the steps of IVF.

\section{Conclusion}

Due to the deficiencies present in conventional IVF procedures, a requirement has emerged for more precise mechanisms. However, based on the literature, MEMS-based systems have proved to be having the potential to overcome those deficiencies and shown an increased rate of success in IVF operations. This article provides a brief introduction to those MEMS-based systems utilized in IVF and identified an abundance of improvements that need to be done for the current systems to be more successful. However, the involvement of the engineering community in ART identified as extremely low. Therefore, this article expects to create interest and inspiration in applying MEMS in IVF.

\section{Acknowledgement}

The authors would like to express their gratitude to the Accelerating Higher Education Expansion and Development (AHEAD) Development Oriented Research (DOR) grant of the Centre for Advanced Mechatronic Systems (CFAMS), University of Moratuwa, Sri Lanka for their valuable advice and guidance towards the success of the research.

\section{Conflicts of Interest}

The authors declare no conflicts of interests.

\section{References}

[1] B. Rizk; J. Garcia-Velasco; H. Sallam; A. Makrigiannakis (Eds.). Infertility and Assisted Reproduction. Cambridge University Press. 2008.

[2] M. Chang. Fertilization of Rabbit Ova in vitro. Nature. 1959, 184, 466-567.

[3] P. C. Steptoe; R. G. Edwards. Birth after the Reimplantation of a Human Embryo. Lancet. 1992, 116, 366.

[4] Z. P. Nagy; A. C. Varghese; A. Agarwal. In Vitro Fertilization. Springer. 2019.

[5] S. C. Esteves, M. Roque, G. Bedoschi, T. Haahr, P. Humaidan. Intracytoplasmic Sperm Injection for Male Infertility and Consequences for Offspring. Nat. Rev. Urol. 2018, 15, 535-562. 
[6] R. M. Kamel. Assisted Reproductive Technology After the Birth of Louise Brown. J Reprod. Infertil. 2013, 14, 96-109.

[7] M. Meseguer; U. Kruhne; S. Laursen. Full in Vitro Fertilization Laboratory Mechanization: Toward Robotic Assisted Reproduction? Fertil. Steril. 2012, 97, 1277-1286.

[8] Strouse. 4 Key Materials When Building Microfluidic Devices. [Online]. Available: https://www.strouse.com/blog/microfluidic-devices.

[9] Applying MEMS Technology to the Diagnosis of Glaucoma. MEMS Invest. [Online]. Available: https://www.memsjournal.com/2009/02/applying-memstechnology-to-the-diagnosis-of-glaucoma-.html.

[10] F. Tools. FT-G Microgripper. [Online]. Available: https://www.femtotools.com/ previousproducts/ft-g-microgripper.

[11] J. W. Judy. Microelectromechanical Systems (MEMS): Fabrication, Design and Applications. Smart Mater. Struct. 2001, 10, 1115-1134.

[12] T. Velten; H. H. Ruf; D. Barrow; N. Aspragathos; P. Lazarou; E. Jung. Packaging of bio-MEMS: Strategies, Technologies, and Applications. IEEE Trans. Adv. Packag. 2005, 28, 533-546.

[13] H. J. Lee; N. Choi; E. S. Yoon; I. J. Cho. MEMS Devices for Drug Delivery. Adv. Drug. Deliv. Rev. 2018; 128, 132-147.

[14] K. J. Rebello. Applications of MEMS in Surgery. Proceedings of the IEEE. 2004, 92, 43-55.

[15] P. Pan; W. Wang; C. Ru; Y. Sun; X. Liu. MEMS-Based Platforms for Mechanical Manipulation and Characterization of Cells, J. Micromech Microeng. 2017, 27, 123003.

[16] V. Casciani; D. Galliano; J. M. Franasiak; G. Mariani; M. Meseguer. Are We Approaching Automated Assisted Reproductive Technology? Sperm Analysis, Oocyte Manipulation and Insemination. FES Reviews. 2021, 2.

[17] W. Asghar; V. Velasco; J. L. Kingsley; M. S. Shoukat; H. Shafiee; R. M. Anchan; G. L. Mutter; E. Tüzel; U. Demirci. Selection of Functional Human Sperm with Higher DNA Integrity and Fewer Reactive Oxygen Species. Adv. Healthc. Mater. 2014, 3, 1671-1679.

[18] Y. Yan; H. Liu; B. Zhang; R. Liu. A PMMA-Based Microfluidic Device for Human Sperm Evaluation and Screening on Swimming Capability and Swimming Persistence. Micromachines. 2020, 11, 793.

[19] European Society of Human Reproduction and Embryology. D. Cytoplasmic Features. [Online]. Available: http://atlas.eshre.eu/es/14555410849002110.

[20] P. Patrizio; E. Fragouli; V. Bianchi; A. Borini; D. Wells. Molecular Methods for Selection of the Ideal Oocyte. Reprod. Biomed. Online. 2007, 15, 346-353.

[21] M. Boukallel; M. Gauthier; M. Dauge; E. Piat; J. Abadie. Smart Microrobots for Mechanical Cell Characterization and Cell Convoying. IEEE Trans. Biomed. Eng. 2007, 54, 1536-1540.

[22] W. Choi; J. S. Kim; D. H. Lee; K. K. Lee; D. B. Koo; J. K. Park. Dielectrophoretic Oocyte Selection Chip for in Vitro Fertilization. Biomed. Microdevices. 2008, 10, 337-345.

[23] M. Hagiwara; T. Kawahara; T. Iijima; F. Arai. High-Speed Magnetic Microrobot 
Actuation in a Microfluidic Chip by a Fine V-Groove Surface. IEEE Trans. Robot. 2013, 29, 363-372.

[24] M. Gauthier; E. Piat. Control of a Particular Micro-Macro Positioning System Applied to Cell Micromanipulation. IEEE Trans. Autom. Sci. Eng. 2006, 3, 264271.

[25] M. Frénéa; S. P. Faure; B. Le Pioufle; P. Coquet; H. Fujita. Positioning Living Cells on a High-Density Electrode Array by Negative Dielectrophoresis. Mater. Sci. Eng. C. 2003, 23, 597-603.

[26] J. E. Swain; D. Lai; S. Takayama; G. D. Smith. Thinking Big by Thinking Small: Application of Microfluidic Technology to Improve ART. Lab Chip. 2013, 13, 1213-1224.

[27] M. Van Den Bergh; E. Bertrand; J. Biramane; Y. Englert. Importance of Breaking a Spermatozoon's Tail before Intracytoplasmic Injection: A Prospective Randomized Trial. Hum. Reprod. 1995, 10, 2819-2820.

[28] C. Leung; Z. Lu; N. Esfandiari; R. Casper; Y. Sun. Automated Sperm Immobilization for Human ICSI. IEEE Trans. Biomed. Eng. 2011, 58, 935-942.

[29] Z. Zhang; C. Dai; J. Huang; X. Wang; J. Liu; C. Ru; H. Pu; S. Xie; J. Zhang; S. Moskovtsev; C. Librach; K. Jarvi; Y. Sun. Robotic Immobilization of Motile Sperm for Clinical Intracytoplasmic Sperm Injection. IEEE Trans. Biomed. Eng. 2019, 66, 444-452.

[30] W. Wang; X. Liu; D. Gelinas; B. Ciruna; Y. Sun. A Fully Automated Robotic System for Microinjection of Zebrafish Embryos. PLoS One. 2007, 2, e862.

[31] Z. Wang; C. Feng; W. T. Ang; S. Y. M. Tan; W. T. Latt. Autofocusing and Polar Body Detection in Automated Cell Manipulation. IEEE Trans. Biomed. Eng. 2017, 64, 1099-1105.

[32] A. M. Hajiyavand; M. Saadat; A. P. S. Bedi. Polar Body Detection for ICSI Cell Manipulation. International Conference on Manipulation, Automation and Robotics at Small Scales (MARSS). 2016, 1-6.

[33] G. D. Palermo; E. S. Sills (Eds.). Intracytoplasmic sperm injection. Springer. 2018.

[34] Z. Lu; X. Zhang; C. Leung; N. Esfandiari; R. F. Casper; Y. Sun. Robotic ICSI (Intracytoplasmic Sperm Injection). IEEE Trans. Biomed. Eng. 2011, 58, 2102-2108.

[35] S. Cui; W. T. Ang. Robotic Micromanipulation of Biological Cells with Friction Force-Based Rotation Control. International Conference on Intelligent Robots and Systems (IROS). 2020, 2792-2798.

[36] Z. Lu; X. Liu; X. Zhang; C. Leung; N. Esfandiari; R. F. Casper; Y. Sun. Automated Robotic Intracytoplasmic Sperm Injection, Pract. Man. Vitr. Fertil. 2012, 353-359.

[37] H. Huang; J. K. Mills; C. Lu; D. Sun. A Universal Piezo-Driven Ultrasonic Cell Microinjection System. Biomed. Microdevices. 2011, 13, 743-752.

[38] K. Sakaki; N. Dechev; R. D. Burke; E. J. Park. Development of an Autonomous Biological Cell Manipulator with Single-Cell Electroporation and Visual Servoing Capabilities. IEEE Trans. Biomed. Eng. 2009, 56, 2064-2074.

[39] X. Li; G. Zong; S. Bi; W. Zhao. Automatic Micromanipulating System for Biological Applications with Visual Servo Control. J Micromech. 2002, 1, 345-363.

[40] Prime Tech Ltd. Piezo-ICSI Standardization Training Manual. 2018. [online]. Available: https://www.youtube.com/watch?v=Jim2TSIItak. 
[41] L. Mattos; E. Grant; R. Thresher; K. Kluckman. New Developments Towards Automated Blastocyst Microinjections. International Conference on Robotics and Automation. 2007, 1924-1929.

[42] H. Matsuoka; T. Komazaki; Y. Mukai; M. Shibusawa; H. Akane; A. Chaki; N. Uetake; M. Saito. High Throughput Easy Microinjection with a Single-Cell Manipulation Supporting Robot. J. Biotechnol. 2005, 116, 185-194.

[43] M. Zareinejad; S. M. Rezaei; A. Abdullah; S. S. Ghidary. Development of a piezoactuated micro-teleoperation system for cell manipulation. Int. J. Med. Robot. 2009, 3, 66-76.

[44] M. Girot; M. Boukallel; S. Régnier. Modeling Soft Contact Mechanism of Biological Cells Using an Atomic Force Bio-Microscope. International Conference on Intelligent Robots and Systems. 2006, 1831-1836.

[45] A. Pillarisetti; M. Pekarev; A. D. Brooks; J. P. Desai. Evaluating the Role of Force Feedback for Biomanipulation Tasks. Symposium on Haptic Interfaces for Virtual Environment and Teleoperator Systems. 2006, 11-18.

[46] D. S. Haliyo; G. Venture. An Overview of the Micro-Manipulation System [mu]MAD. International Conference on Advanced Intelligent Mechatronics. 2005, 390-395.

[47] R. Seifabadi; S. M. Rezaei; S. S. Ghidary; M. Zareinejad. A Teleoperation System for Micro Positioning with Haptic Feedback. Int. J. Control. Autom. Syst. 2013, 11, 768-775.

[48] M. Saadat; A. M. Hajiyavand; A. pal S. Bedi. Oocyte Positional Recognition for Automatic Manipulation in ICSI. Micromachines. 2018, 9, 429.

[49] X. Liu; Z. Lu; Y. Sun. Orientation Control of Biological Cells under Inverted Microscopy. IEEE/ASME Trans. Mechatronics. 2011, 16, 918-924.

[50] Z. Wang; C. Feng; R. Muruganandam; J. Mathew; P. C. Wong; W. T. Ang; S. Y. M. Tan; W. T. Latt. A fully automated robotic system for three-dimensional cell rotation. International Conference on Robotics and Automation (ICRA). 2016, 1707-1712.

[51] B. Song; Y. Feng; Q. Zhou; L. Feng. On-Chip Three-dimension Cell Rotation Using Whirling Flows Generated by Oscillating Asymmetrical Microstructures. International Conference on Intelligent Robots and Systems (IROS). 2019, 678683.

[52] Y. Yalikun; Y. Aishan; A. Mosha; K. Sumiyama; Y. Tanaka. Oocyte All-Surfaces' Imaging Method Using Micro-Scale Rotational Flow. Micro Nano Lett. 2018, 13, 306-311.

[53] M. C. Wu. Optoelectronic Tweezers. Nat. Photonics. 2011, 5, 322-324.

[54] S. K. Mohanty; P. K. Gupta. Laser-Assisted Three-Dimensional Rotation of Microscopic Objects. Rev. Sci. Instrum. 2004, 75, 2320-2322.

[55] D. Meyer; M. L. P. Colon; H. V. Alizadeh; L. Su; B. Behr; D. B. Camarillo. Orienting oocytes using vibrations for in-vitro fertilization procedures, International Conference on Robotics and Automation (ICRA). 2019, 4837-4843.

[56] L. Feng; B. Song; Y. Chen; S. Liang; Y. Dai; Q. Zhou; D. Chen; X. Bai; Y. Feng; Y. Jiang; D. Zhang; F. Arai. On-Chip Rotational Manipulation of Microbeads and Oocytes Using Acoustic Microstreaming Generated by Oscillating Asymmetrical 
Microstructures. Biomicrofluidics. 2019, 13, 064103.

[57] Y. Eguchi; M. Ogiue-Ikeda; S. Ueno. Control of Orientation of Rat Schwann Cells Using an 8-T Static Magnetic Field. Neurosci. Lett. 2003, 351, 130-132.

[58] P. Benhal; J. G. Chase; P. Gaynor; B. Oback; W. Wang. AC Electric Field Induced Dipole-Based on-Chip 3D Cell Rotation. Lab. Chip. 2014, 14, 2717-2727.

[59] J. L. Sebastián; S. Muñoz; M. Sancho; G. Martínez; G. Álvarez. Electromechanical Effects on Multilayered Cells in Nonuniform Rotating Fields. Phys. Rev. E. 2011, 84, 011926 .

[60] D. S. Gray; J. L. Tan; J. Voldman; C. S. Chen. Dielectrophoretic Registration of Living Cells to a Microelectrode Array. Biosens. Bioelectron. 2004, 19, 1765-1774.

[61] S. Zappe; M. Fish; M. P. Scott; O. Solgaard. Automated MEMS-Based Drosophila Embryo Injection System for High-Throughput RNAi Screens. Lab. Chip. 2006, 6, 1012-1019.

[62] M. S. Watanabe; N. Kakuta; K. Mabuchi; Y. Yamada. Micro-thermocouple probe for measurement of cellular thermal responses, Engineering in Medicine and Biology 27th Annual Conference. 2005, 4858-4861.

[63] D. F. Miller; S. L. Holtzman; T. C. Kaufman. Customized Microinjection Glass Capillary Needles for P-Element Transformations in Drosophila Melanogaster. Biotechniques. 2002, 33, 366-372.

[64] A. Gilani; J. Koohsorkhi; A. Shamloo. Novel Method for Cell Penetration Based on MEMS Technology with Failure and Impacting Characterization of Mouse Oocyte Membrane. Sens. Actuator A Phys. 2017, 263, 461-470.

[65] W. Johnson; C. Dai; J. Liu; X. Wang; D. K. Luu; Z. Zhang; C. Ru; C. Zhou; M. Tan; H. Pu; S. Xie; Y. Peng; J. Luo; Y. Sun. A Flexure-Guided Piezo Drill for Penetrating the Zona Pellucida of Mammalian Oocytes. IEEE Trans. Biomed. Eng. 2018, 65, 678-686.

[66] N. Okuyama; R. Obata; N. Oka; N. Aono; T. Hashimoto; K. Kyono. The Clinical Results of Piezo-ICSI Compared to Conventional-ICSI: A Sibling-Oocyte Study. Fertil. Steril. 2019, 112.

[67] Y. Fujii; Y. Endo; S. Mitsuhata; M. Hayashi; H. Motoyama. Evaluation of the Effect of Piezo-Intracytoplasmic Sperm Injection on the Laboratory, Clinical, and Neonatal Outcomes. Reprod. Med. Biol. 2020, 19, 198-205. 